\title{
Refusal to renew an expiring protocol
}

"I'm really sorry, Connie, but I don't have the authority to extend your protocol's expiration date. The NIH/OLAW policy is pretty clear about that. The IACUC sent you three notices that it was nearing its expiration date, but a renewal application was never received. In the meantime, you can work on your other protocols, but this one is now a holding protocol. You have to stop all work on this protocol until you get it renewed."

"You must be kidding, Larry," was the response from Dr. Connie Linder. "We're talking about a week, maybe less, and you're making me jump through bureaucratic hoops and stop funded research?"

"I understand why you're upset," answered Larry Covelli, the IACUC Chair, "but you're the one who stopped it. The IACUC has no choice in this. The IACUC office will send you a letter saying you have 30 days to submit a new protocol, and during that time, Lab Animal Resources will care for your animals, but no research or breeding can be done, and you're going to have to pay for the care of the animals."

"And what happens if I don't submit one in 30 days?" queried Linder.

The answer came slowly but clearly: "The animals are now under a holding protocol, with the IACUC as the investigator. Nothing will be done other than providing husbandry and any needed medical care. The IACUC will determine their fate, which can include euthanasia, donation to other investigators, etc."

Hearing that, Linder hung up her phone and complained, to no avail, to all of her colleagues.

Thirty days later, there still was no protocol renewal from Linder. Covelli was unsure what to do, because in similar past instances, the investigators had always submitted their protocol renewals as quickly as possible. Covelli decided to send an ultimatum to Linder, in which he warned her that if a protocol renewal application was not received within 30 days, the 172 mice that were on the holding protocol would be euthanized. Thirty days passed again with no renewal submission. Covelli finally discussed the issue with the IACUC, and the committee's decision was to euthanize the animals if other investigators could not use them. That was not what Covelli wanted to hear, although he didn't know what he did want to hear.

Do you think that the IACUC's initial action and Covelli's subsequent actions were ethical and permissible under policies of the National Institutes of Health and Office of Laboratory Animal Welfare? How would you approach the problems faced by Covelli?

\section{RESPONSE}

\section{Cruel to be kind}

\section{Alison D. Pohl, MS, RLATG, CPIA \& Ron G. Wallace, PhD, CIH, RBP}

Both the Chair (Covelli) and the IACUC were caught unprepared. This situation may have been avoided by having a cohesive Animal Care Program supported by IACUC policies and procedures. The principal investigator (PI) was given adequate warnings that her protocol was expiring, but the Chair and IACUC didn't know what to do if the warnings went unheeded. When people don't know what to do, particularly in high-pressure situations, they may improvise in ways that are counterproductive.

Other than sending the warning letters, the uninformed IACUC seems to have taken no initial actions. Covelli's initial action of granting 30 days to submit a protocol application was acceptable; however, additional actions should have been taken at that time, such as securing the animals, notifying the Grants Office and setting up weekly reminders to the PI. Because a renewal protocol was never submitted, one wonders whether Linder, acting in bad faith, might have just finished off the week's work she needed to do after the expiration of her protocol. This argues that animals on holding protocols should be secured or at least closely monitored. In addition, the IACUC should have been notified at the point of expiration that the PI was no longer authorized to use the animals.

The Chair's actions in granting the second 30-day period seem to be compliant with both the Public Health Service Policy on Humane Care and Use of Laboratory Animals ${ }^{1}$ and the Animal Welfare Act and Regulations ${ }^{2}$ because the animals from the expired protocol were covered under an approved holding protocol. But his actions seem to have exacerbated the problem. Granting the second 30-day period, without
IACUC involvement, sets a bad precedent and weakens the perceived authority of the IACUC. Attention needs to be paid to a nuance like this where 'public' perception is involved. In the end, the Animal Care Program is hurt by the Chair's attempt to soften the blow to the PI. Ethically, the Chair is caught between helping the PI and upholding institutional standards.

This situation should have been addressed programmatically. PIs should be trained to understand that IACUCapproved protocols cannot be extended and must not lapse unless there are extenuating circumstances; that they will not be able to work with their animals after the protocol expires; and that expenses for the animals during that period of time cannot be supported by the PI's Public Health Service funding (i.e., funds used during periods of non-compliance have to be reimbursed to the federal source, which involves further unpleasant procedures between the institution and the PI). 